\title{
A combination of the preoperative neutrophil-to- lymphocyte and lymphocyte-to-monocyte ratios as a useful predictor of survival outcomes following the transarterial chemoembolization of huge hepatocellular carcinoma
}

\author{
Juanfang Liu, PhD, Wenguang Zhang, MD, Rongfang Niu, MM, Yahua Li, PhD, Xueliang Zhou, PhD, Xinwei Han, PhD.
}

\begin{abstract}

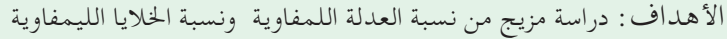

(NLR)

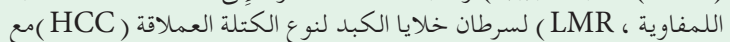

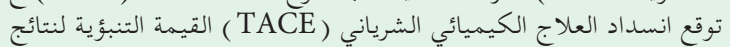
بقاء المريض.

المنهجية : تحليل بأثر رجعي من 180 مريضاً يعانون من سرطان خلايا الكبد

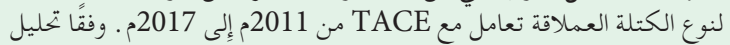

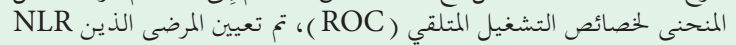

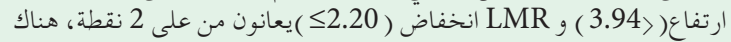

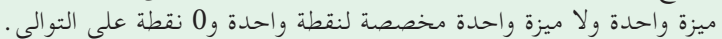

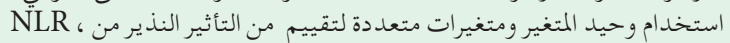

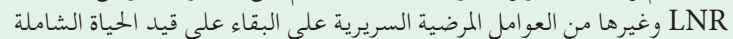

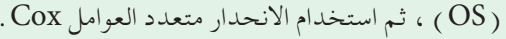

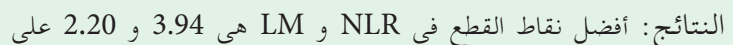

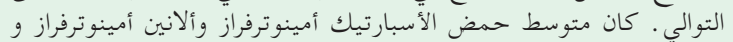

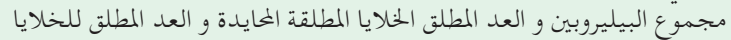

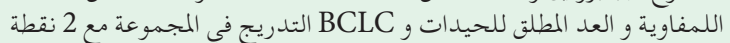

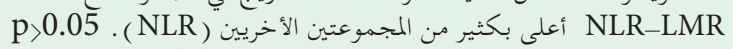

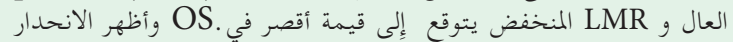

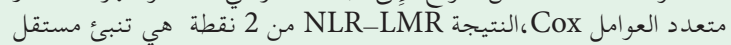

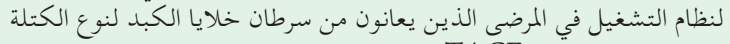

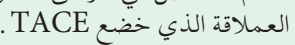

الخلاصة : LMR و NLR قبل العلاج هو تنبئ بسيطة وفعالة لنتائج البقاء

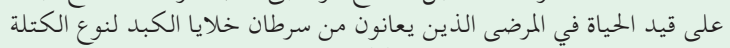

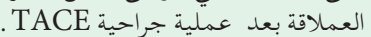

Objectives: To investigate the prognostic value of neutrophil-to-lymphocyte ratio (NLR) plus the lymphocyte-to-monocyte ratio (LMR) to predict survival outcomes in huge hepatocellular carcinoma (HCC) patients undergoing transarterial chemoembolization (TACE).

Methods: There were 180 huge HCC patients undergoing TACE between 2011 and 2017 were retrospectively analyzed. Patients who has an increased NLR (>3.94) and a decreased LMR $(\leq 2.20)$ were assessed score 2 according to receiver operating characteristic (ROC) curve, and patients who were assigned with 1 , with one of these characteristic or 0 with neither of these characteristics. We used univariate and multivariate analyses for evaluations of the predicative NLR, LMR and other values about overall survival (OS) using multivariate Cox's regression.

Results: The liver function index such as aspartate transaminase, alanine transaminase, and total bilirubin, as well as inflammatory biomarkers like absolute neutrophil count, monocyte count, lymphocyte count, seemed much larger than the groups with an NLR-LMR score of 2 than in the other 2 groups $(p<0.05$ for all), including BCLC stage. Higher NLR plus a low level of LMR predicted a short median OS. Multivariate Cox's regression revealed that an NLR-LMR score of 2 was a useful predictor of OS in huge HCC patients after TACE.

Conclusion: The pretreatment NLR plus LMR are effective for predicting survival outcomes in huge HCC patients after TACE.

Keywords: Neutrophil-to-lymphocyte ratio, lymphocyte-to-monocyte ratio, huge hepatocellular carcinoma

Saudi Med J 2020; Vol. 41 (4): 376-382 doi: 10.15537/smj.2020.4.24911

From the Department of Interventional Radiology, The First Affiliated Hospital of Zhengzhou University, Zhengzhou, China.

Received 31st July 2019. Accepted 6th January 2020.

Address correspondence and reprint request to: Dr. Xinwei Han, Department of Interventional Radiology, The First Affiliated Hospital of Zhengzhou University, Zhengzhou, China. E-mail:1021056466@qq.com

ORCID ID: https://orcid.org/0000-0003-0564-7949 
A $s$ the sixth most frequent neoplasm as well as the third leading cause of cancer death worldwide, hepatocellular carcinoma (HCC) is one of the most common aggressive malignancies. Its incidence is expected to rise later. ${ }^{1}$ Huge $\mathrm{HCC}$ is defined as HCC with a nodule larger than $10 \mathrm{~cm}$. Huge HCC is regularly combined with other prognostic factors such as macro/microvascular invasion or the presence of multiple tumors. Lots of treatments are not feasible, but transcatheter arterial chemoembolization (TACE) can improve the control of intrahepatic recurrence and prognosis. ${ }^{2,3}$ However, some patients with huge HCC gain benefits from TACE. Hence, differentiating the patients benefiting from TACE from patients not benefiting from it. Previous studies demonstrated that inflammation-based markers like the neutrophil-tolymphocyte ratio (NLR), prognostic nutritional index (PNI), LMR and albumin-bilirubin grade (ALBI) do a good job in the progress of cancer in patients with HCC. ${ }^{4-7} \mathrm{Chu}$ et $\mathrm{al}^{8}$ reported that the pretreatment NLR and PNI are convenient and helpful forecasters of overall survival (OS) in HCC patients after RFA therapy. Combining NLR and PLR was validated to predict recurrence and prediction of HCC in patients undergoing RFA. ' Recently, studies proved that LMR is a superior predictor of OS than established biomarkers. ${ }^{10,11}$ A high NLR was related to bad prognosis in patients with HCC underwent TACE. ${ }^{12}$ On the other hand, a high preoperative LMR predicted a favorable OS and progress-free survival (PFS). ${ }^{11}$ Yang et $\mathrm{al}^{10}$ showed that the LMR and NLR were useful inflammation-based forecasters in predicting of tumor recurrence in HCC patients undergoing hepatic resection. Recently, the combination of the NLR and the LMR for patients with HCC after TACE has not been developed. This study aim to determine whether a combination of NLR and LMR can help predict the outcomes of TACE-treated patients with huge HCC.

Methods. Between January 2011 and May 2017, 180 patients with huge HCC experiencing TACE in our hospital have been retrospectively included. This study was approved by the institutional review board (IRB) of The First Affiliated Hospital of Zhengzhou University, Zhengzhou, People's Republic of China and was conducted according to the principles expressed in

Disclosure. Authors have no conflict of interests, and the work was not supported or funded by any drug company. the Declaration of Helsinki and informed consent was obtained from all participants before TACE.

All patients with primary HCC according to the American Association for the Study of Liver Diseases (AASLD) guidelines, ${ }^{13}$ and TACE was recommended as the best treatment by a multidisciplinary team for patients not eligible for other treatments including aggressive surgery, liver transplantation, and tumor ablation. They must meet the requirements: i) nodules with a diameter larger than $10 \mathrm{~cm}$. ii) no other simultaneous malignancies, and an iii) Eastern Cooperative Oncology Group (ECOG) Performance Status score of 0 or 1 . They should not have any of the below symptoms: severe dysfunction of the lung, heart, kidney, and so forth; coagulation abnormity; acute inflammatory phase; and active concomitant infection. Patients who received percutaneous ablation, particle implantation, partial liver resection or other therapies before or after TACE treatment were excluded from this study as well.

Transcatheter arterial chemoembolization procedure. After a successful Seldinger puncture in the right groin, a 5-F introducer sheath was positioned. Selective catheterization of the target artery has been used to determine the supply of blood for tumors. Then, super selective catheterization of the feeding arteries was achieved using a microcatheter (Merit Maestro, Merit Medical System, Inc., USA). Subsequently, a mixture of ethiodized oil with pirarubicin (THP, 20-40 mg, Jiangsu, China) was administered as a chemoembolic material to occlude the feeding arteries of the tumor. The embolization endpoint was the stasis of blood flow; when this was not achieved, gelatin sponges were injected to obtain complete stasis. All the procedures in this study will be recorded.

Follow-up. Thirty days later after the experiment, participants were followed up by blood testing and imageological examination of contrast enhanced CT or enhanced MRI, with the aim of evaluating the tumor reaction and to decide individual treatment plans. Three months later, CT or MRI was carried out once more to judge tumor progression after TACE procedure. The number of treatments ranged from one to many based on multiple factors including the health of the patient, hepatic capacity, tumor reappearance and other blood testing. The data of participants were recorded until death occurred or the cutoff date (October 31, 2018). The endpoints of the study were tumor recurrence at 3 months after the first TACE procedure. The modified Response Evaluation Criteria in Solid Tumors $(\mathrm{mRECIST})^{14}$ has been used to evaluate patients' 
reaction after receiving the treatment. The OS time ranged from the first TACE to the death. Progressionfree survival (PFS) refers to the time from the first TACE to that of disease progression or death.

Data collection and evaluation. One to 3 days before the TACE, blood testing were taken. Neutrophilto-lymphocyte ratio has been considered as the total neutrophil calculation under the standard of lymphocyte calculation. Moreover, LMR has been assessed based on divided lymphocyte count and monocyte count. The ideal cutoff of NLR and PLR values were derived from the curve analysis of receiver operating characteristic (ROC).

Statistical analysis. The optimum preoperative NLR and LMR cutoff values were derived from a ROC curve. Mean \pm standard deviation is used to present continuous variables and one-way analysis were compared between participants. Pearson $x^{2}$ test or Fisher's exact test were utilized to categorize data expressed as frequencies or proportions. The OS was obtained with the method of the Kaplan-Meier, and OS times were calculated by the test of log-rank. The factors of various dangers for OS have been figured out with multivariate Cox's proportional hazard regression analysis. A $p<0.05$ displayed the significance of the statistics. Every analysis of statistics was finished by IBM SPSS Statistics for Windows, version 10 (IBM Corp., Armonk, NY., USA).

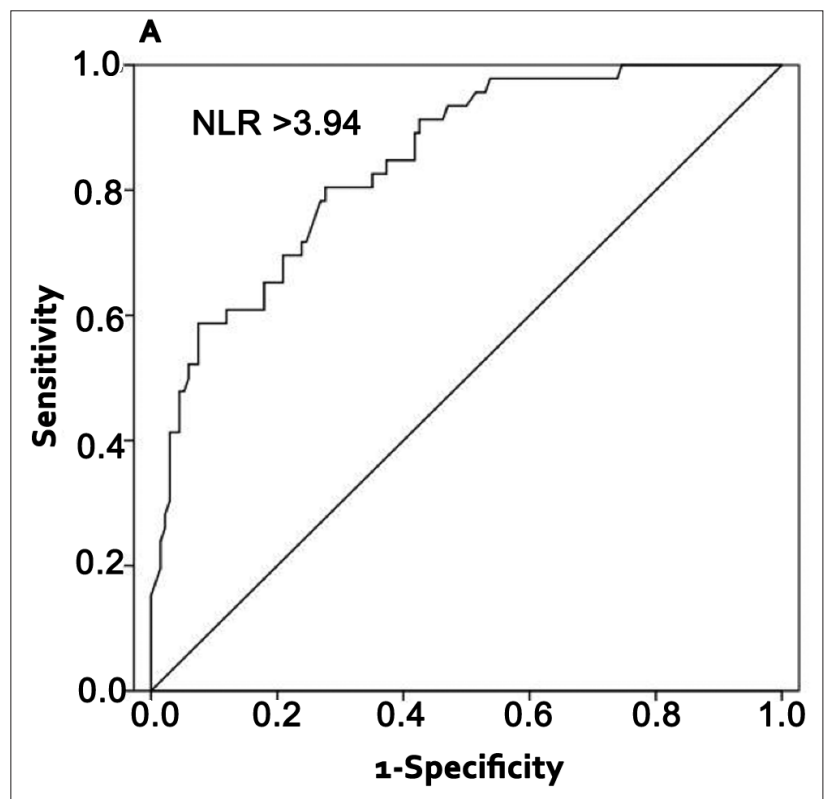

Results. Optimal cutoff values for the NLR and the LMR. Tumor progression was assessed 3 months after the first TACE procedure, and we stratified each prognostic index based on the peak and cutoff points of ROC curve. Neutrophil-to-lymphocyte ratio ideal cutoff value should be 3.94 whose sensitivity was 0.804 and a specificity of 0.724 (AUROC curve: 0.845; 95\% CI: 0.782-0.907; $p<0.001$ ) (Figure 1A). The ideal cutoff value for the LMR was 2.20 whose sensitivity was 0.604 and specificity 0.87 (AUROC curve: 0.751 ; 95\%: 0.676-0.825; $p<0.001$ ) (Figure 1B). The purposes is to estimate the score of NLR-LMR individually using these values. Accordingly, the patients belong to 3 groups exhibited in Table 1.

Baseline characteristics. The study group included 155 male and 25 female with an average age of $54.3 \pm 9.3$ years. The average size of tumors was 12.1 \pm 1.9 (range $10.0-18.8 \mathrm{~cm})$, and $103(57.2 \%)$ patients had portal vein thrombosis. Overall, the mean TACE treatments times was $2.43 \pm 1.13$ (range: $1-7$ ).

Associations between NLR-LMR grade and clinicopathologic characteristics of the HCC patients. Among the 180 included patients, $82(45.6 \%)$ patients were assigned to the NLR-LMR 0 group, 29 (16.1\%) patients were assigned to the NLR-LMR 1 group, and $69(38.3 \%)$ patients were assigned to the NLR-LMR 2 group. These 3 groups did not show great differences regarding their genders, ages, and the sizes of tumors. The hepatic function, inflammatory biomarkers

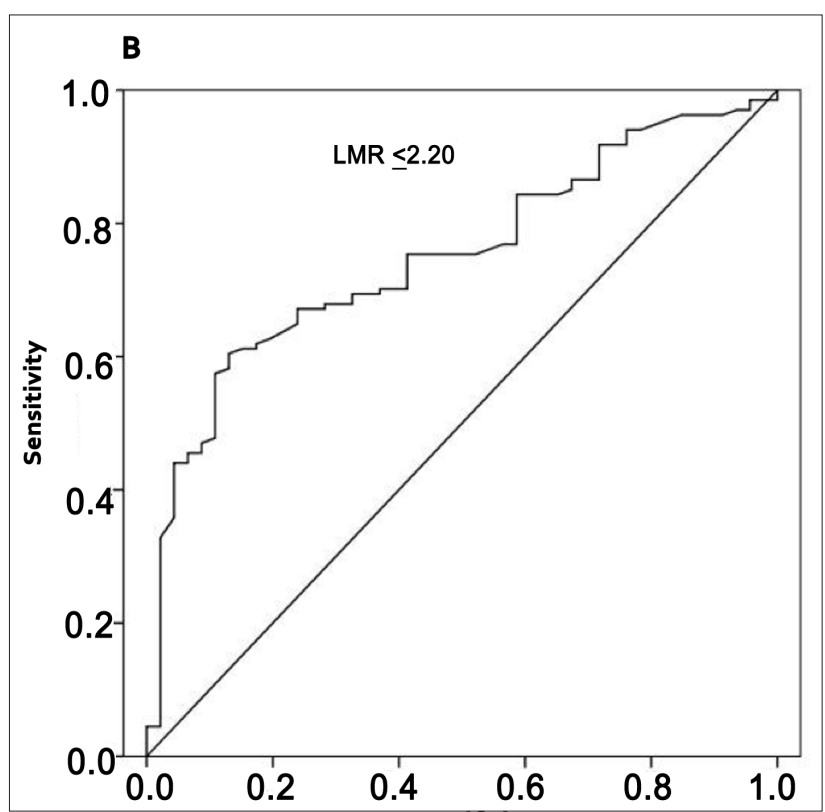

Figure 1 - Receiver operating characteristics curves to assess the best cutoff value of the neutrophil-to-lymphocyte ratio (NLR) and lymphocyte-tomonocyte ratio (LMR) . A) receiver operating characteristic (ROC) curve of the NLR. B) ROC curve of the LMR. 
and BCLC stage were considerably elevated in the NLR-LMR group 2 than that of groups 1 or 3 ( $p<0.05$ for all). Detailed information of the 3 groups are summarize in Table 2.

Survival analysis. Among the patients, a total of 175 (97.2\%) has been dead during the recording period. And the median OS has been 8.7 months (range, 3.9-32 months). Using the Kaplan-Meier analysis, it has been found that median OS for participants (NLR $\leq 3.94$ ) was longer than that for participants (NLR >3.94) $(8.6$ months versus 7.5 months, $p<0.001$ ) (Figure 2B). The higher the NLR was, the lower the OS for patients would be. Likewise, median OS for participants with an LMR >2.2 has been registered higher than that for participants whose LMR was lower (Figure 2C). The higher the LMR was, the longer the OS in huge HCC patients after TACE treatment. Finally, patients with NLR-LMR scores of 2 had the worst outcomes as their median OS was only 7.0 months, compared with participants in the NLR-LMR 1 (median OS 8.7 months) and NLR-LMR 0 (median OS 10.4 months) groups $(p<0.05)$ (Figure 2D).

Risk factors for outcome after TACE. As shown in Table 3, tumor size, Child-pugh stage, PVTT, NLR, LMR, NLR-LMR score, and BCLC stage have much to do with a short OS. In addition, factors below can predict OS independently using multivariate analysis
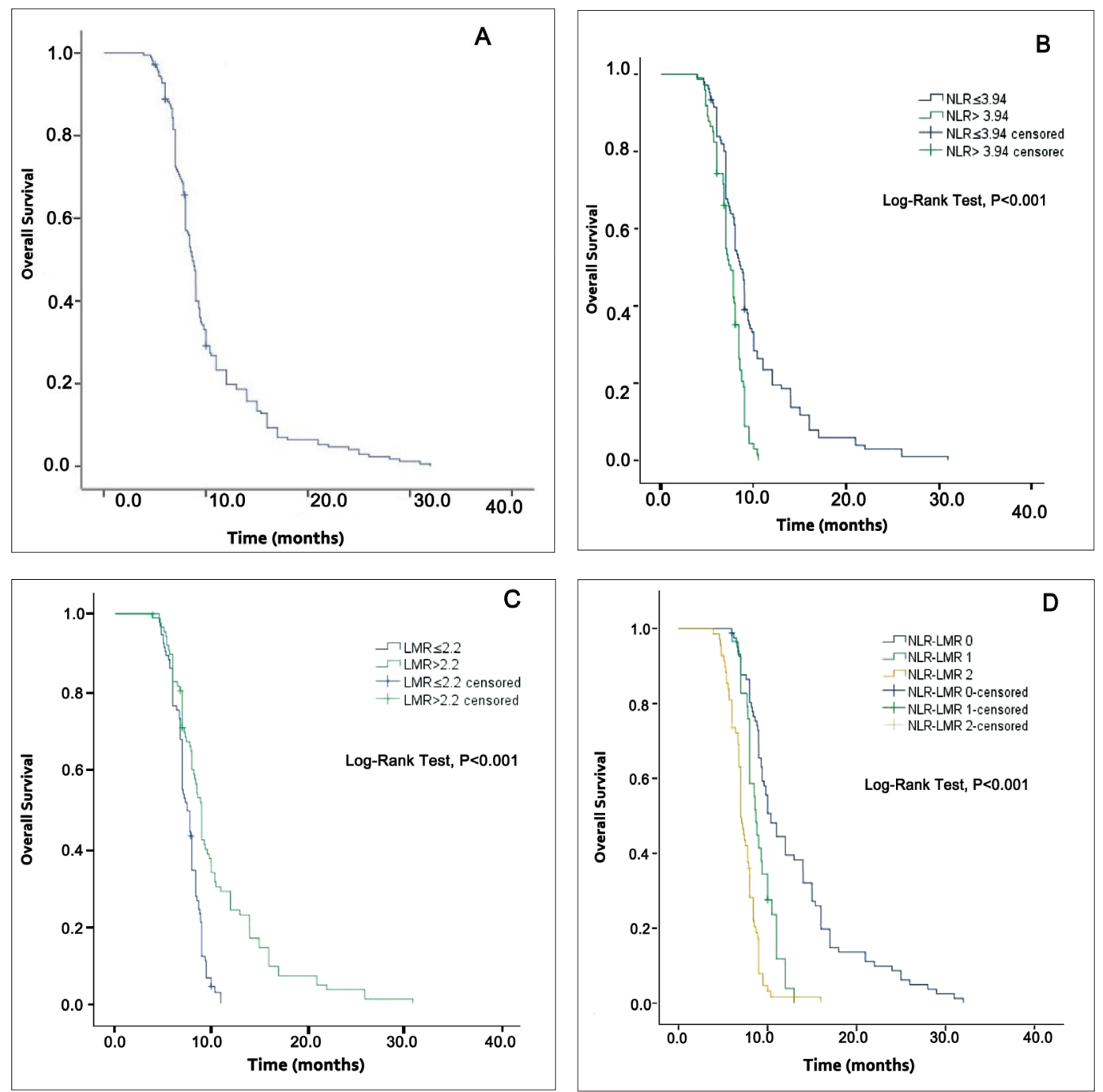

Figure 1 - Kaplan-Meier survival curves for overall survival in huge hepatocellular carcinoma patients who underwent transarterial chemoembolization. A) overall survival, B) the neutrophil-to-lymphocyte ratio (NLR), C) the lymphocyte-to-monocyte ratio (LRM) and D) the NLR-LMR score. 
of Cox's regression: Child-Pugh stage $(p<0.001)$, tumor size $(p=0.006)$, and an NLR-LMR score of $2(p=0.014)$.

Discussion. The NLR has been demonstrated as a prognostic serum biomarker for many malignancies. ${ }^{15-17}$ Larger HCC is often associated with a high NLR. ${ }^{18}$ Neutrophil-to-lymphocyte ratio cutoff values vary between different medical centers and different populations, so there is no optimal NLR for all patients. ${ }^{9,12,19}$ In this case, the best NLR cutoff value

Table 1 - The combination of the neutrophilto-lymphocyte ratio (NLR) and the lymphocyte-to-monocyte ratio (LMR) as prognostic indices.

\begin{tabular}{lc}
\hline Variables & Score \\
\hline$N L R$ & \\
$<3.94$ & 0 \\
$\geq 3.94$ & 1 \\
$L M R$ & \\
$<2.20$ & 1 \\
$\geq 2.20$ & 0 \\
$N L R-L M R$ & \\
$N L R=0$ and $L M R=0$ & 0 \\
$N L R=1$ or $L M R=1$ & 1 \\
$N L R=1$ and $L M R=1$ & 2 \\
\hline
\end{tabular}

suitable for huge HCC patients were selected by ROC curve analysis. In this study, participants whose NLR is larger than 3.94 showed a considerably poorer OS than the others. These results were coincident in former findings, in which a high preoperative NLR had something to do with shorter OS in patients after TACE treatment for unresectable HCC. ${ }^{6,20}$ Lymphocytes are essential parts of human immune system. Fewer lymphocytes indicates an aberrant protected mechanism and a weakening in body immunity. For this reason, elevated levels of neutrophils or decreased levels of lymphocytes lead to an increased NLR and thus a worse prognosis in cancer patients. The LMR as a novel prognostic indicator has been proven to helpful to forecast the therapeutic effects for participant with HCC when experiencing the surgical resection. ${ }^{11}$ Additionally, there is no unified, standard optimal cutoff value for the LMR. The curve analysis of ROC was applied in order to examine the ideal LMR cutoff value in this research. It has been mentioned that those with NLR $>2.2$ displayed OS of longer time than those having LMR $\leq 2.2$ in terms of univariate analysis. These results shared some similarities with what have been found before, in which a high preoperative LMR score was related to long OS in HCC patients. ${ }^{11,21}$ The mechanism by which a decreased LMR indicates short survival time is not very clear, but some studies have provided some possible interpretations. Lymphocytes

Table 2 - A comparison of the clinical characteristics of patients with different NLR-LMR scores.

\begin{tabular}{|c|c|c|c|c|}
\hline Variables & $\begin{array}{c}\text { NLR-LMR } 0 \\
(\mathrm{n}=82)\end{array}$ & $\begin{array}{c}\text { NLR-LMR } 1 \\
(\mathrm{n}=29)\end{array}$ & $\begin{array}{c}\text { NLR-LMR } 2 \\
(\mathrm{n}=69)\end{array}$ & $P$-value \\
\hline Age (years) $($ mean $\pm S D)$ & $54.5 \pm 9.6$ & $55.7 \pm 9.5$ & $53.4 \pm 9.0$ & 0.511 \\
\hline Gender (male/female) & $72 / 10$ & $22 / 7$ & $61 / 8$ & 0.218 \\
\hline HBsAg (positive/negative) & $70 / 12$ & $23 / 6$ & $63 / 6$ & 0.251 \\
\hline Tumor size $($ mean $\pm S D)$ & $117.9 \pm 17.6$ & $124.6 \pm 23.4$ & $123.6 \pm 18.5$ & 0.113 \\
\hline Child-Pugh stage (A/B) & $66 / 16$ & $17 / 12$ & $39 / 30$ & 0.004 \\
\hline PVTT (yes/no) & $32 / 50$ & $19 / 10$ & $52 / 17$ & 0.018 \\
\hline BCLC stage $(\mathrm{B} / \mathrm{C})$ & $67 / 15$ & $15 / 14$ & $19 / 50$ & $<0.001^{*}$ \\
\hline Absolute neutrophil count $($ mean $\pm S D)$ & $3.69 \pm 1.9$ & $4.3 \pm 1.8$ & $4.9 \pm 1.9$ & $0.002^{\dagger}$ \\
\hline Absolute lymphocyte count $($ mean $\pm S D)$ & $1.6 \pm 0.8$ & $1.2 \pm 0.4$ & $0.8 \pm 0.3$ & $<0.001^{*}$ \\
\hline Absolute monocyte count $($ mean $\pm S D)$ & $0.5 \pm 0.3$ & $0.8 \pm 0.4$ & $0.7 \pm 0.3$ & $<0.001^{\dagger}$ \\
\hline Abumin-bilirubin $($ mean $\pm S D)$ & $35.6 \pm 4.5$ & $34.8 \pm 6.7$ & $36.2 \pm 5.1$ & 0.284 \\
\hline TBIL $(\mathrm{mmol} / \mathrm{L})(\operatorname{mean} \pm \mathrm{SD})$ & $15.1 \pm 7.4$ & $17.7 \pm 9.1$ & $27.4 \pm 13.8$ & $<0.001^{\dagger}$ \\
\hline $\operatorname{ALT}(\mathrm{IU} / \mathrm{L})(\mathrm{mean} \pm \mathrm{SD})$ & $43.5 \pm 23.5$ & $53.3 \pm 23.9$ & $70.4 \pm 57.9$ & $<0.001^{\dagger}$ \\
\hline $\operatorname{AST}(\mathrm{IU} / \mathrm{L})(\operatorname{mean} \pm \mathrm{SD})$ & $36.1 \pm 16.7$ & $39.9 \pm 19.6$ & $58.8 \pm 37.1$ & $<0.001^{\dagger}$ \\
\hline TACE (number of times; mean $\pm \mathrm{SD}$ ) & $2.6 \pm 1.1$ & $2.3 \pm 1.5$ & $2.2 \pm 0.9$ & 0.122 \\
\hline
\end{tabular}


Table 3 - Prognostic factors related to overall survival (OS) .

\begin{tabular}{|c|c|c|c|c|c|c|}
\hline Variables & HR & \multicolumn{2}{|l|}{ Univariate } & \multicolumn{2}{|l|}{ Multivariate } & $P$-value \\
\hline Age $(\leq 55$ vs $>55)$ & 1.159 & $(0.859-1.565)$ & 0.335 & - & - & - \\
\hline Gender (male vs female) & 1.308 & $(0.837-2.044)$ & 0.253 & - & - & - \\
\hline Tumor size ( $>12 \mathrm{vs}<12 \mathrm{~cm}$ ) & 1.765 & $(1.304-2.388)$ & $<0.001$ & 1.575 & $(1.139-2.177)$ & 0.006 \\
\hline Barcelona Clinic Liver Cancer stage (C vs B) & 3.251 & $(2.307-4.581)$ & $<0.001$ & - & - & - \\
\hline Child-Pugh stage (B vs A) & 3.374 & $(2.352-4.839)$ & $<0.001$ & 2.401 & $(1.624-3.548)$ & $<0.001$ \\
\hline PVTT (Yes vs No) & 1.589 & $(1.174-2.149)$ & 0.003 & - & - & - \\
\hline $\operatorname{NLR}(>3.94$ vs $\leq 3.94)$ & 3.694 & $(2.620-5.209)$ & $<0.001$ & - & - & - \\
\hline $\operatorname{LMR}(\leq 2.2$ vs $>2.2)$ & 3.541 & $(2.512-4.994)$ & $<0.001$ & - & - & - \\
\hline NLR-LMR score & & & & - & - & - \\
\hline 0 & & & & - & - & - \\
\hline 1 & 2.263 & $(1.426-3.594)$ & 0.001 & - & - & - \\
\hline 2 & 4.999 & $(3.405-7.339)$ & $<0.001$ & 3.797 & $(1.306-11.04)$ & 0.014 \\
\hline
\end{tabular}

and monocytes are the fundamental constituents of human antitumor immunity. However, monocytosis weakens the antitumor functioning of the system of immunity and promotes how the tumors come up and develop. ${ }^{22}$ Hence, a decreased LMR reflects the impairment of host immune surveillance. In our study, we found that a high preoperative NLR and a low LMR (NLR-LMR 2) were closely related to some factors: a high AST level, high ALT level, high total bilirubin (TBIL) concentration, elevated neutrophil count, elevated monocyte count, decreased lymphocyte count, portal vein tumor thrombus (PVTT), large tumor as well as a late BCLC stage. The predictive NLR-LMR value for huge HCC participants who underwent TACE was confirmed. A high NLR-LMR score were related to a short OS in patients with HCC. These findings are in accordance with the results of an accumulating number of studies. ${ }^{8,10,21}$ In combined with the results of this study, huge HCC patients may benefit from the preoperative NLR-LMR score as a valuable prognostic indicator in the near future. Some undeniable restricts are shown in this research. To begin with, the nature of the study is retrospective, and there may have been selective bias in data selection and analysis. Second, the number of patients is not much. Therefore, it is urgent to carry out prospective multicenter studies to prove the forecast value of the NLR plus LMR.

In conclusion, our single-center study revealed that pretreatment NLR-LMR score was important to predict OS patients with huge HCC receiving TACE treatment. The NLR-LMR score can be a new inflammatory biomarker for predicting the outcomes of participants whose HCC was huge with TACE.
Acknowledgment. We would like to thanks the American Journal Experts for English editing.

\section{References}

1. Forner A, Reig M, Bruix J. Hepatocellular carcinoma. The Lancet 2018; 391: 1301-1314.

2. Wakayama K, Kamiyama T, Yokoo H, Orimo T, Shimada $S$, Einama T, et al. Huge hepatocellular carcinoma greater than $10 \mathrm{~cm}$ in diameter worsens prognosis by causing distant recurrence after curative resection. Journal of Surgical Oncology 2017; 115: 324-329.

3. Meniconi RL, Komatsu S, Perdigao F, Boelle PY, Soubrane O, Scatton O. Recurrent hepatocellular carcinoma: a Western strategy that emphasizes the impact of pathologic profile of the first resection. Surgery 2015; 157: 454-462.

4. Wu SJ, Lin YX, Ye H, Li FY, Xiong XZ, Cheng NS. Lymphocyte to monocyte ratio and prognostic nutritional index predict survival outcomes of hepatitis B virus-associated hepatocellular carcinoma patients after curative hepatectomy. J Surg Oncol 2016; 114: 202-210.

5. Liu C, Li L, Lu WS, Du H, Yan LN, Yang JY, et al. Neutrophillymphocyte ratio plus prognostic nutritional index predicts the outcomes of patients with unresectable hepatocellular carcinoma after transarterial chemoembolization. Scientific Reports 2017; 7: 13873.

6. Rice J, Dodge JL, Bambha KM, Bajaj JS, Reddy KR, Gralla J, et al. Neutrophil-to-lymphocyte ratio associates independently with mortality in hospitalized patients with cirrhosis. Clinical gastroenterology and hepatology : the official clinical practice Clin Gastroenterol Hepatol 2018; 16: 1786-1791.e1.

7. Li C, Zhang XY, Peng W, Wen TF, Yan LN, Li B, et al. Preoperative albumin-bilirubin grade plus platelet-tolymphocyte ratio predict the outcomes of patients with BCLC stage A hepatocellular carcinoma after liver resection. Medicine 2018; 97: e11599.

8. Chu MO, Shen $\mathrm{CH}$, Chang TS, Xu HW, Yen CW, Lu SN, et al. Pretreatment Inflammation-Based Markers Predict Survival Outcomes in Patients with Early Stage Hepatocellular Carcinoma After Radiofrequency Ablation. Scientific Reports 2018; 8: 16611 . 
9. Chen K, Zhan MX, Hu BS, Li Y, He X, Fu SR, et al. Combination of the neutrophil to lymphocyte ratio and the platelet to lymphocyte ratio as a useful predictor for recurrence following radiofrequency ablation of hepatocellular carcinoma. Oncol Lett 2018;15: 315-323.

10. Yang YT, Jiang JH, Yang HJ, Wu ZJ, Xiao ZM, Xiang BD. The lymphocyte-to-monocyte ratio is a superior predictor of overall survival compared to established biomarkers in HCC patients undergoing liver resection. Sci Rep 2018; 8: 2535.

11. Lin ZX, Ruan DY, Li Y, Wu DH, Ma XK, Chen J, et al. Lymphocyte-to-monocyte ratio predicts survival of patients with hepatocellular carcinoma after curative resection. World J Gastroenterol 2015; 21: 10898-10906.

12. Liu C, Jia BS, Zou BW, Du H, Yan LN, Yang JY, et al. Neutrophilto-lymphocyte and aspartate-to-alanine aminotransferase ratios predict hepatocellular carcinoma prognosis after transarterial embolization. Medicine 2017; 96: e8512.

13. Heimbach J, Kulik L, Finn R, Sirlin C, Abecassis M, Roberts $\mathrm{L}$, et al. AASLD guidelines for the treatment of hepatocellular carcinoma. Hepatology 2018; 67: 358-380.

14. Seyal AR, Gonzalez-Guindalini FD, Arslanoglu A, Harmath CB, Lewandowski RJ, Salem R, et al. Reproducibility of mRECIST in assessing response to transarterial radioembolization therapy in hepatocellular carcinoma. Hepatology 2015; 62: 1111-1121.

15. Semeniuk-Wojtas A, Lubas A, Stec R, Syrylo T, Niemczyk S, Szczylik C. Neutrophil-to-lymphocyte ratio, platelet-tolymphocyte ratio, and C-reactive protein as new and simple prognostic factors in patients with metastatic renal cell cancer treated with tyrosine kinase inhibitors: A systemic review and meta-analysis. Clin Genitourin Cancer 2018; 16: e685-e693.
16. Chen G, Zhu L, Yang Y, Long Y, Li X, Wang Y. Prognostic Role of Neutrophil to Lymphocyte Ratio in Ovarian Cancer: A Meta-Analysis. Technol Cancer Res Treat 2018; 17: 1533033818791500 .

17. Hu J, Wang N, Yang Y, Ma L, Han R, Zhang W, et al. Diagnostic value of alpha-fetoprotein combined with neutrophil-to-lymphocyte ratio for hepatocellular carcinoma. BMC Gastroenterol 2018; 18: 186.

18. Llovet JM, Bruix J. Novel advancements in the management of hepatocellular carcinoma in 2008. J Hepatol 2008; 48 Suppl 1: S20-S37.

19. Arai K, Fukumoto T, Kido M, Tanaka M, Kuramitsu K, Kinoshita $\mathrm{H}$, et al. Preoperative neutrophil-to-lymphocyte ratio as a predictor of survival after reductive surgery plus percutaneous isolated hepatic perfusion for hepatocellular carcinoma: a retrospective analysis. Surg Today 2017; 47: 385-392.

20. Zhou D, Liang J, Xu LI, He F, Zhou Z, Zhang Y, et al. Derived neutrophil to lymphocyte ratio predicts prognosis for patients with HBV-associated hepatocellular carcinoma following transarterial chemoembolization. Oncol Lett 2016; 11: 2987-2994.

21. Mano Y, Yoshizumi T, Yugawa K, Ohira M, Motomura T, Toshima T, et al. Lymphocyte-to-monocyte ratio is a predictor of survival after liver transplantation for hepatocellular carcinoma. Liver Transpl 2018; 24: 1603-1611.

22. Mano Y, Aishima S, Fujita N, Tanaka Y, Kubo Y, Motomura $\mathrm{T}$, et al. Tumor-associated macrophage promotes tumor progression via STAT3 signaling in hepatocellular carcinoma. Pathobiology 2013; 80: 146-154. 